\title{
Editorial
}

\section{New Initiative: “Ten Questions” Paper Series in Building \& Environment}

\author{
Bert Blocken $^{\mathrm{a}, \mathrm{b}} *$ \\ ${ }^{a}$ Department of the Built Environment, Eindhoven University of Technology, P.O. Box 513, 5600 Eindhoven, The \\ Netherlands \\ ${ }^{b}$ Department of Civil Engineering, Leuven University, Kasteelpark Arenberg 40 - Bus 2447, 3001 Leuven, Belgium
}

\section{Brief description of the initiative}

Building \& Environment is launching a new initiative for a series of "Ten Questions" papers, to be published in a pre-described format. "Ten Questions" papers should deal with a well-defined topic and should be centered around a selection of ten relevant and topical questions. Submissions are based on invitation only by the Editor of the Special Initiative (SI), Prof. Bert Blocken. Papers will be recruited in a way that ensures balanced coverage of all topics in the scope of Building and Environment. The papers should be (co-)authored by established researchers in the field with proven expertise on the topic, typically by an extensive track record of previously published books and/or peer-reviewed journal papers. The questions are selected and answered by the author(s). The questions should be such that the paper is visionary, authoritative and can provide younger researchers directions for future research.

The submission and review procedure is outlined in detail in section 2 below.

Building \& Environment intends to publish annually at least 12 papers in this series, where this paper will be published as the first paper in every volume. Depending on the number of accepted "Ten Questions" papers, the publication scheme can be adjusted to include more or less of these papers in a volume.

\section{Submission and review procedure}

“Ten Questions" papers will undergo a two-step review process.

\section{STEP 1}

Invited authors will submit the following components of the intended paper to the Editor of the SI by email to b.j.e.blocken@tue.nl:

- title

- abstract (max. 250 words)

- keywords

- tentative list of ten questions

\footnotetext{
* Corresponding author: Bert Blocken, Building Physics and Services, Eindhoven University of Technology, P.O. Box 513, 5600 MB Eindhoven, the Netherlands. Tel.: +31 (0)40 247 2138, Fax +31 (0)40 2438595

E-mail address: b.j.e.blocken@tue.nl
} 
- one-sentence answers to the questions

- description of expertise of the authors on the topic (max. 250 words)

- list of five suggested reviewers (name, title, affiliation, email address, reason for selection)

- intended submission date of full paper

The title of every paper should be similar and should start with the words: "Ten questions concerning ...". Two examples:

- "Ten questions concerning CFD simulation of pollutant dispersion around buildings"

- "Ten questions concerning thermal comfort in office buildings"

The questions should be as short and as clear as possible. The preferred question length is below 10 words. The maximum question length is 15 words. The Editor of the SI will evaluate whether the proposed contribution fits the scope and requirements of the series of "Ten Questions" papers and whether it does not provide unwanted overlap with previously published review or other papers in Building and Environment or other journals. If evaluated positively, the Editor of the SI will already select three expert reviewers, some of which can be taken from the list of five suggested reviewers. The reviewers will remain anonymous. The reviewers are welcome to improve the formulation of the questions or to suggest some other questions for consideration by the author(s). The same reviewers will afterwards evaluate the full paper. This first and preliminary review stage is handled outside the Elsevier Editorial System (EES). It should help minimizing the effort by the author(s) in revising the full paper afterwards. The author(s) will subsequently send the revised set of components to the Editor of the SI (again by email to b.j.e.blocken@tue.nl) together with a detailed response to reviewers, addressing every point raised by them.

\section{STEP 2}

Following approval of the revised set of components by the Editor of the SI, the authors will prepare the full paper that should not exceed 8000 words. The structure of every "Ten Questions" paper is given below. The maximum number of figures is 15 . The introduction should address the reasons for selecting these specific questions. The answers to the questions should satisfy the requirements of original review or other papers. Appropriate referencing should be used. Authors should take care to include not only their own work but also relevant work by others in the answers to the questions. New research results cannot be introduced in the position paper, as this is not the purpose of this type of paper and its limited length does not allow full description of the research that has led to the new results. Where the author(s) provide their personal view on the topic without support by literature references, this should be clearly indicated as such. The biography section should indicate the expertise of the authors on the topic of the paper. Apart from the above-mentioned requirements, the "Ten Questions" papers should also adhere to the regular requirements stated in the Building \& Environment "Guide for Authors" on the journal website. The author(s) submit the full paper by means of the EES by selecting the option "Ten Questions" in the submission drop-down list. The paper will be forwarded to the three anonymous expert reviewers that were already selected in the preliminary review stage in step 1 . This second part of the review process is similar to that for regular papers.

\section{Mandatory structure of "Ten Questions" papers:}




\section{Abstract}

Introduction

1. "Question 1: ..."

Answer to question 1

2. "Question 2: ..."

Answer to question 2

3. $\ldots$

4. ...

10. "Question 10: ..."

Answer to question 10

\section{Conclusions}

Acknowledgements

Biography

References 